\section{Phosphopeptide vaccines for cancer}

\section{By Lev Osherovich, Senior Writer}

U.S. researchers have overcome a roadblock to the use of phosphorylated peptides as a way to improve the specificity and potency of therapeutic cancer vaccines, according to a paper in the Proceedings of the National Academy of Sciences of the USA. ${ }^{1}$ The approach, which employs phosphopeptides derived from tumor-associated proteins, could complement the strategy of Cytos Biotechnology AG, which is in Phase II testing with a melanoma vaccine derived from one of the proteins in the study.

Cancer vaccines, including Cytos', are typically designed to evoke cytotoxic T lymphocyte (CTL) responses. The new study shows that phosphorylated peptides can activate helper T cells upstream of CTLs and thus magnify the immune response to cancer antigens.

Phosphorylated peptides should make especially good cancer vaccine candidates because they are found in cancerous cells but not in normal cells. Many proteins in tumor cells are overphosphorylated compared with normal cells, due to hyperactivation of proliferationassociated kinases.

"Phosphopeptides represent a new cohort of tumor antigens recognized by the immune system," said Suzanne Topalian, professor of surgery and oncology at Johns Hopkins University. "Because increased expression of intracellular phosphoproteins in dysregulated signaling pathways can support the malignant characteristics of tumor cells, phosphopeptides may provide selective targets for immunotherapy."

However, the approach has not yet been pursued because of difficulties in getting immune cells to properly process and present phosphopeptides.

To avoid this problem, a team co-led by Topalian and two University of Virginia researchers-Victor Engelhard, professor of microbiology; and Donald Hunt, professor of chemistry and pathology-has been looking for aberrantly phosphorylated protein fragments that elicit immunity.

In 2006, Engelhard and Hunt's research groups identified phosphorylated proteins on tumor surfaces that interacted with major histocompatibility complex class I (MHC I) proteins, which trigger the CTL response. ${ }^{2}$ In the new study, the team tested whether tumorderived phosphopeptides also could interact with major histocompatibility complex class II (MHC II), which activates the helper T cells that amplify CTL activity.

The researchers isolated phosphopeptides bound to MHC II molecules on the surface of cultured tumor cells and normal cells from two melanoma patients. They found 42 phosphopeptides derived from 21 different proteins that were present in either or both of the two tumor cells lines but not on normal cells.

The group focused on phosphopeptides derived from a well known melanoma-associated protein, melan-A (MLANA; MART1), that were highly abundant in both tumor cell lines.

In cell culture, immune cells from a third melanoma patient were stimulated with a MART1 phosphopeptide, leading to a phosphopeptide-specific helper $\mathrm{T}$ cell response. When cultured with melanoma cells, those helper $\mathrm{T}$ cells responded to the tumor cells by secreting interferon- $\gamma$, a cytokine that stimulates other immune cells. The same T cells did not respond to control cells lacking the phosphopeptide antigen on their surface.

\section{First class response}

Cytos is using an MHC I-selective fragment of MART1 in its CYT004-MelQbG10 melanoma vaccine, where the peptide is conjugated to a ligand for toll-like receptor 9 (TLR9), a component of the innate immune system that increases the peptide's immunogenicity. The company's peptide is derived from a different part of MART1 than the phosphorylated fragment in Topalian's study.

Cytos EVP and CSO Martin Bachmann said that while the company's peptide is already a potent antigen, he thinks a vaccine derived from MART1 phosphopeptides could lead to improved immunological targeting of tumors over normal cells.

"The use of phosphorylated peptides may bring a new level of specificity into the field of cancer vaccines," he said.

In addition, Bachmann said, a phosphopeptide vaccine might delay the emergence of MART1 mutations that help cancers evade the immune system, a phenomenon called antigen escape. The reason, he said, is that phosphopeptides secreted by tumor cells are picked up by nearby antigen-presenting cells and are quickly recognized as abnormal by the immune system, perhaps more efficiently than unphosphorylated protein fragments.

"It may be attractive to combine Cytos' MART1 vaccine with such newly described peptides in order to minimize escape of the tumor," said Bachmann.

Bachmann also suggested that getting a phosphopeptide into the clinic could involve combining the antigen with an adjuvant or a TLR agonist.

Meanwhile, Topalian and colleagues are looking for evidence of immunological responses to MART1 phosphopeptides in other melanoma patients and hope to examine the contribution of phosphopeptides in approved and investigational immunomodulatory therapies for melanoma. 


\section{TARGETS \& MECHANISMS}

Topalian, Engelhard and Hunt have filed a patent on their discoveries, and the IP is available for licensing.

Osherovich, L. SciBX 2(27); doi:10.1038/scibx.2009.1099

Published online July 23, 2009

\section{REFERENCES}

1. Deponteiu, F. et al. Proc. Natl. Acad. Sci. USA; published online July 6 , 2009; doi:10.1073/pnas.0903852106

Contact: Suzanne L. Topalian, The Johns Hopkins University School of
Medicine, Baltimore, Md.

e-mail: stopali1@jhmi.edu

Contact: Donald F. Hunt, University of Virginia, Charlottesville, Va. e-mail: dfh@virginia.edu

2. Zarling, A.L. et al. Proc. Natl. Acad. Sci. USA 103, 14889-14894 (2006)

COMPANIES AND INSTITUTIONS MENTIONED

Cytos Biotechnology AG (SIX:CYTN), Zurich, Switzerland

Johns Hopkins University, Baltimore, Md.

University of Virginia, Charlottesville, Va. 